\title{
Study of Elastic Behavior of Metallic Thin Films by 2D Synchrotron XRD and in situ Tensile Testing
}

Geandier Guillaume, Renault Pierre-Olivier, Goudeau Philippe, Eric Le Bourhis, and Girault Baptiste

Laboratoire de Métallurgie Physique, University of Poitiers, UMR 6630 CNRS, SP2MI-Téléport 2-Bd Marie et Pierre Curie, BP 30179, Futuroscope-chasseneuil Cedex, F-86962, France

\begin{abstract}
Understanding the mechanical behavior of nano-structured thin films in relation to their structure, in particular to the grain size, is of high importance for the development of technological applications. In this work, model nanometric multilayer W/Au systems exhibiting different structures are elaborated. These films are supported by a (thin) polyimide substrate. Films mechanical response is characterized experimentally by tensile tests carried out in-situ in a $\mathrm{X}$-ray diffractometer set on a synchrotron source. Transmission X-ray diffraction has been used to study the deformations of both $\mathrm{W}$ and Au sublayers as a function of the applied load. This geometry has been developed in the aim of optimizing the experiment time.
\end{abstract}

\section{INTRODUCTION}

For a few years, there has been an increasing interest in the elastic properties of thin films [1-4]. Literature data seem to show that the elastic behavior of metallic thin films can differ significantly from their bulk counterparts because of their specific structure (texture, defects, high density of interfaces, interface mixing) and of size effects [5-8]. Studying and tailoring the size effect on elastic constants of polycrystalline thin films require controlling the structure (grain size, residual stresses, and texture). One way to control grain size along one direction at nanometric scales is to prepare multilayers.

Elastic behavior of supported thin films can be determined by combining tensile test [9-11] or four point bending [12-14] and X-ray diffraction (XRD). X-ray diffraction is phase selective and hence is a unique technique that allows determination of both the mechanical and structural states of the diffracting phases. This technique can be used to study how the elastic constants are affected by the period thickness independently of the multilayer components. The main disadvantage of the XRD technique is that the X-ray measurements are time consuming for thin multilayers (as the diffracting volume is quite small) even for high flux synchrotron radiation sources.

The aim of this paper is to show how the elastic behavior of multilayers can be studied from the in situ loading of the specimen during the X-ray diffraction experiment. We report a tensile test study of W/Au multilayers films deposited on kapton substrate. X-ray strain measurements with relevant precision being time consuming, measurements were performed using synchrotron $\mathrm{X}$ ray diffraction combined with a $2 \mathrm{D}$ detector in order to increase the amount of data which improves the analysis precision [15] as compared to usual point detector (0D) analysis [16]. 


\section{SAMPLES}

The substrates used to support the multilayered films were $127.5 \mu \mathrm{m}$ thick kapton dogbone substrates; the in-plane sample dimensions were $14 \times 6 \mathrm{~mm}^{2}$. The substrate was cleaned with ethanol before deposition. Films were deposited by ion beam sputtering. The base pressure in the growth chamber was $7 \times 10^{-5} \mathrm{~Pa}$ while the working pressure during film growth was approximately $10^{-2} \mathrm{~Pa}$. To obtain multilayered films, $\mathrm{W}$ and $\mathrm{Au}$ are deposed sequentially; the period (thickness of $\mathrm{W}$ plus $\mathrm{Au}$ layers) has been calibrated before the experiment on silicon substrates. One layer is constituted of an equal thickness of $\mathrm{W}$ and $\mathrm{Au}$, the gas pressure in the chamber being chosen to obtain stress free samples. The total thickness was measured by a Dektak II-a surface profilometer system, to be $640 \pm 10 \mathrm{~nm}, 750 \pm 10 \mathrm{~nm}$ and $720 \pm 10 \mathrm{~nm}$ for the $12 \mathrm{~nm}, 6 \mathrm{~nm}$ and $3 \mathrm{~nm}$ period films respectively.

\section{EXPERIMENTAL METHOD}

Experiments have been conducted on the 11.3.1 beamline at the Advanced Light Source (Berlekey, USA). The samples have been mounted perpendicularly to the beam direction on the Deben testing module; special mounting grips have been used to achieve this geometry. A Si powder has been placed on the sample surface, at the measuring point in order to correct the detector orthogonality and the sample movement during loading. Energy was set at $10 \mathrm{keV}$ to get the maximum number of photons and a clear signal from the two phases. The detector has been set at a distance of $90 \mathrm{~mm}$ from the sample, to capture a major part of the Si $\{220\}$ planes reflection. The geometry of the experiment is described on Figure 1.

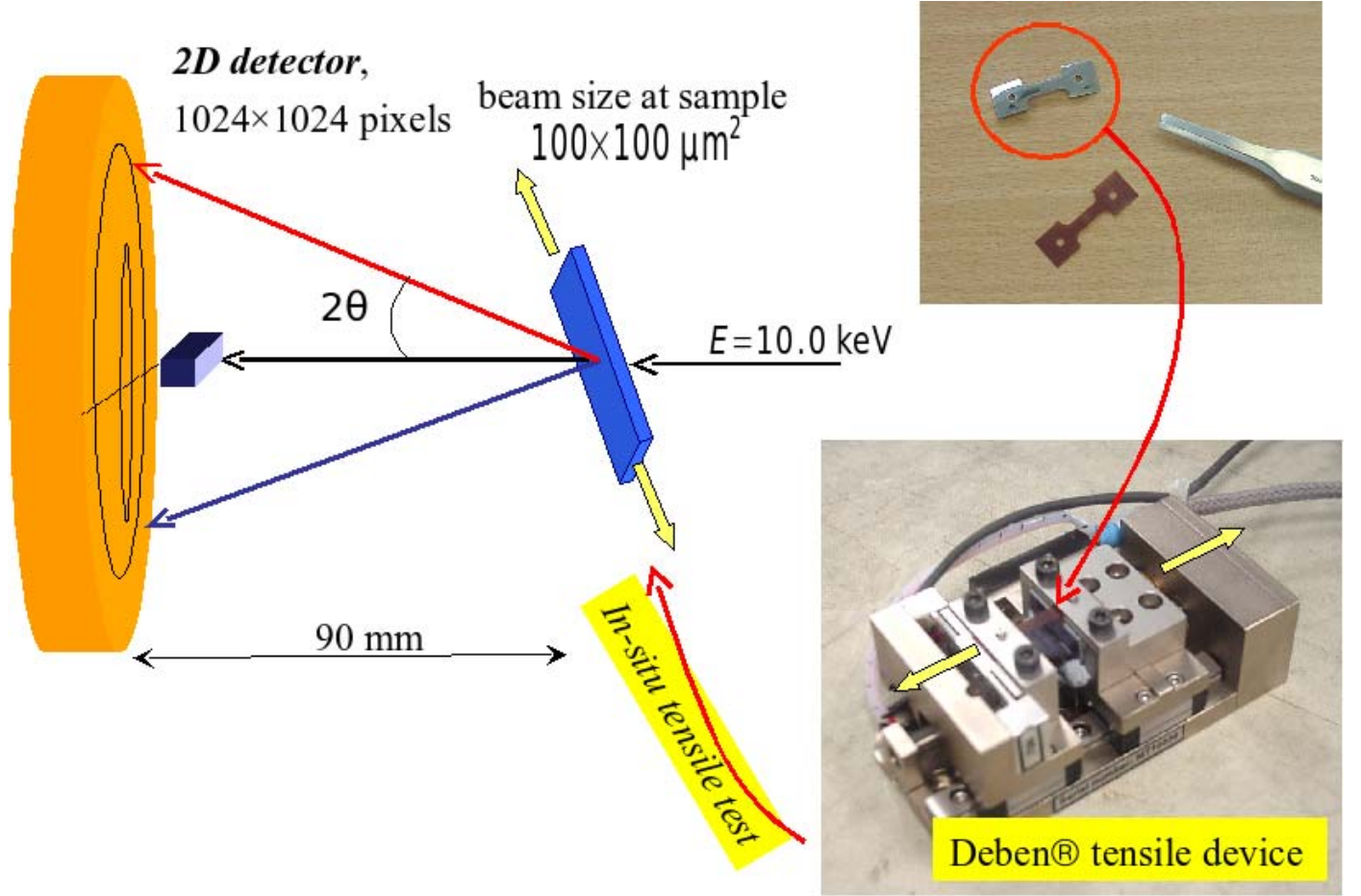

Figure 1. Experimental setup on the 11.3.1 beamline.

Due to the films texture, only the strongest peaks ( $\{110\}$ for $\mathrm{W}$ and $\{111\}$ for Au) will be 
recorded during the experiments. Gold $\{220\}$ peaks are detected on the images, but their intensity is too low to determine the strains precisely. It is possible to increase the intensity of the $\{220\}$ gold peak by tilting the sample according to the beam direction. This rotation tends to increase the intensity of the $\{111\}$ and $\{220\}$ peaks on one part of the image, but to decrease their intensity on the other part. On the same image, we have the diffracted signals from the gold and the tungsten phases and from the silicon powder (Figure 2).

The transmission geometry has been chosen to optimize the acquisition rate, every image being recorded with an exposure time of $60 \mathrm{~s}$. With such a short exposure time, dynamic loading of the sample has been performed with loading rates ranging between $0.01 \mathrm{~N}$ and $0.5 \mathrm{~N}$ per minute.

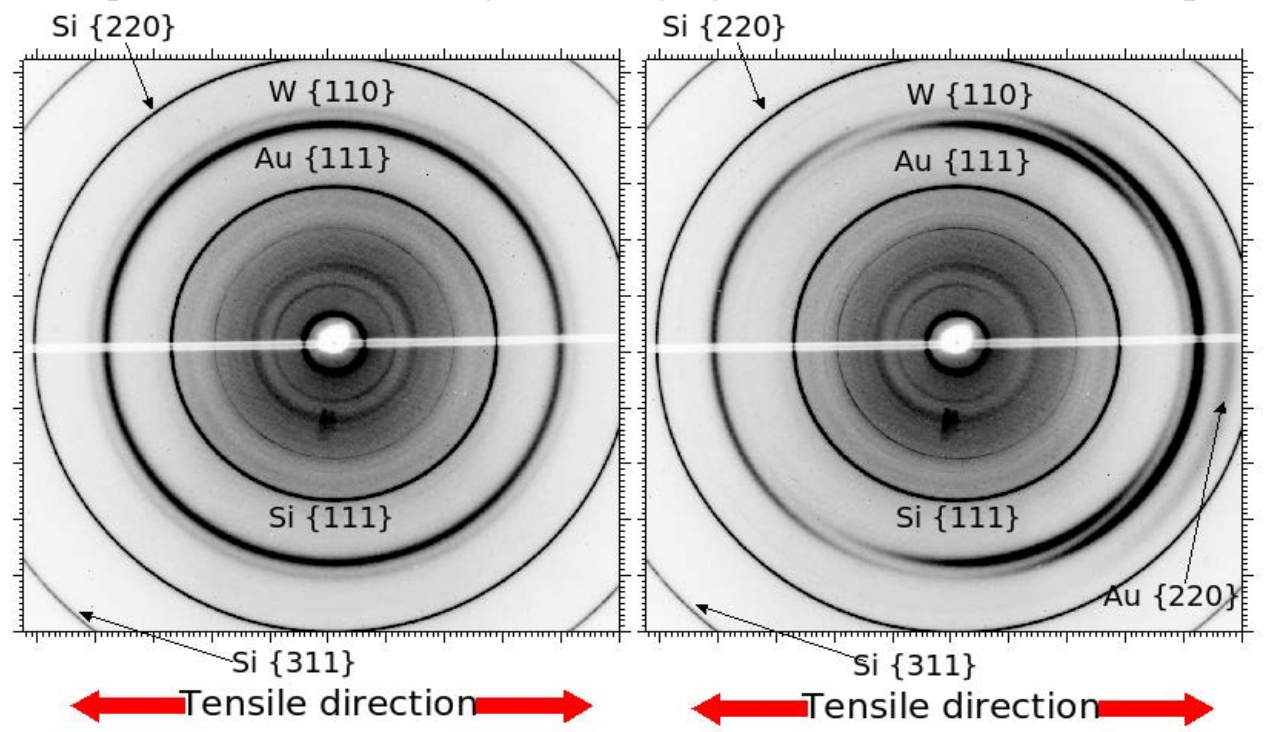

Figure 2. Example of signals recorded on the W/Au sample with a period of $12 \mathrm{~nm}$ (left: without sample rotation, right: with a sample rotation of $15^{\circ}$ ).

\section{TRANSMISSION GEOMETRY AND DYNAMICAL LOADING}

The sample is loaded under a constant rate. Figure 3 presents the evolution of the applied load as a function of time as recorded by the Deben module. Points where images are recorded for the $12 \mathrm{~nm}$ period sample are also indicated on this graph. On the figure, Deben time is the load-time curve as recorded by Deben software and image time is the recorded time saved in each image header. Each image is an accumulation of the diffracting signal during $60 \mathrm{~s}$, and is thus an average image over the related load variation. We can note that the complete experiment can be done in only a few hours. For example, using the sample made of a $12 \mathrm{~nm}$ period W/Au multilayer with a rotation of $15^{\circ}$ and a rate of $0.035 \mathrm{~N}$ per minute, 163 images were recorded between 0.5 and $12.5 \mathrm{~N}$ (entire experiment duration is less than 7 hours).

\section{RESULTS AND DISCUSSION}

Images are corrected for geometrical aberrations by the dedicated Bruker software and converted into tiff format to be read by Fit2d analysis software[17] which is used to calibrate and to reduce the data. Specific codes are used to prepare the reduced data for subsequent fitting by GNUplot software.

As the silicon powder and W/Au signals are recorded at the same time, Si powder rings are used 
to calibrate the geometrical parameters of the experiment, assuming that the x-ray beam wavelength is stable. Sample to detector distance as well as image center and detector non orthogonality are calibrated. Using the calibrated geometry parameters (center, distance and tilt angles) the data were divided into $1^{\circ}$ bins $(\Delta \varphi)$ and integrated azimuthally in a process referred to as 'caking' in the Fit2d lexicon. Reduced data are saved to obtain, for each load step, classical $2 \theta$ intensity diagrams, one for each bin (360 diagrams per load step). Specific code is used to generate input for GNUplot in order to fit the Si peaks that are complete over the ring and W $\{110\}$ and $\mathrm{Au}\{111\}$ peaks. The code allows for locating the peak, and extracting its parameters, adjusting a Pearson VII function with an iteration process. Keeping $\varphi$ constant, parameters from one adjustment are passed to the next load step in the GNUplot script. Parameters extracted from each peak adjustment are: the position, intensity, FWHM, M coefficient of the Pearson VII function and background polynomial forms (which can be of degrees 0 to 6 , but linear background has been used). At the end of this procedure, we have, at each loading step and for each $\varphi$ positions (360 in total) all the parameters for the W, Au and Si peaks.

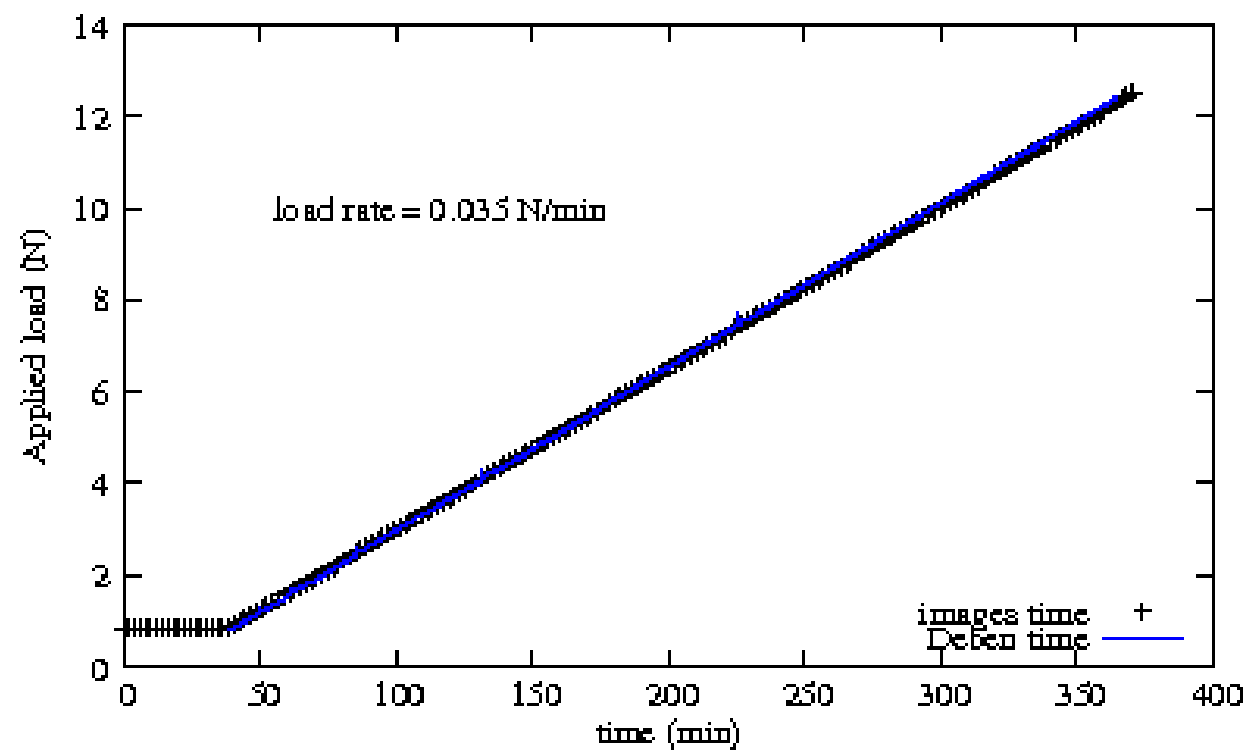

Figure 3. Evolution of the applied load as a function of time for the $12 \mathrm{~nm}$ period W/Au sample

Strains in each phase ( $\mathrm{Si}, \mathrm{W}$ and $\mathrm{Au}$ ) can be determined using the following rational definition:

$$
\varepsilon=\ln \left(\frac{\sin \theta_{0}}{\sin \theta}\right)
$$

where $\theta_{0}$ is the peak position at the reference load (here the first step), so that relative strains due to external loading and not residual strains are determined.

Figure 4 presents the strain evolution in the tungsten layer as a function of the applied load for a $15^{\circ}$ rotation of the sample. Due to the sample rotation, all $\varphi$ positions cannot be analyzed. On figure 4, x-ray strain in both phases with common $\varphi$ positions are presented. Along the tensile direction $\left(\varphi\right.$ positions around $90^{\circ}$ and $270^{\circ}$ ), the layer is under tensile stress while along the transversal direction $\left(\varphi\right.$ positions around $0^{\circ}$ and $\left.180^{\circ}\right)$, the layer is under compressive stress. At the end of the loading cycle, tungsten response presents strain saturation over a load of $10 \mathrm{~N}$, probably because of the onset of plasticity in one of the two layers (probably the Au layers where similar behavior is obtained [18]). Work is in progress to determine diffraction peak broadening 
over plastic threshold in both phases in order to confirm this hypothesis (onset plasticity in gold). For the $12 \mathrm{~nm}$ sample, X-rays diffraction allows following simultaneously the behaviors of both $\mathrm{Au}$ and $\mathrm{W}$ phases in the multilayer film, and the peaks for each phase can be adjusted without major problems.

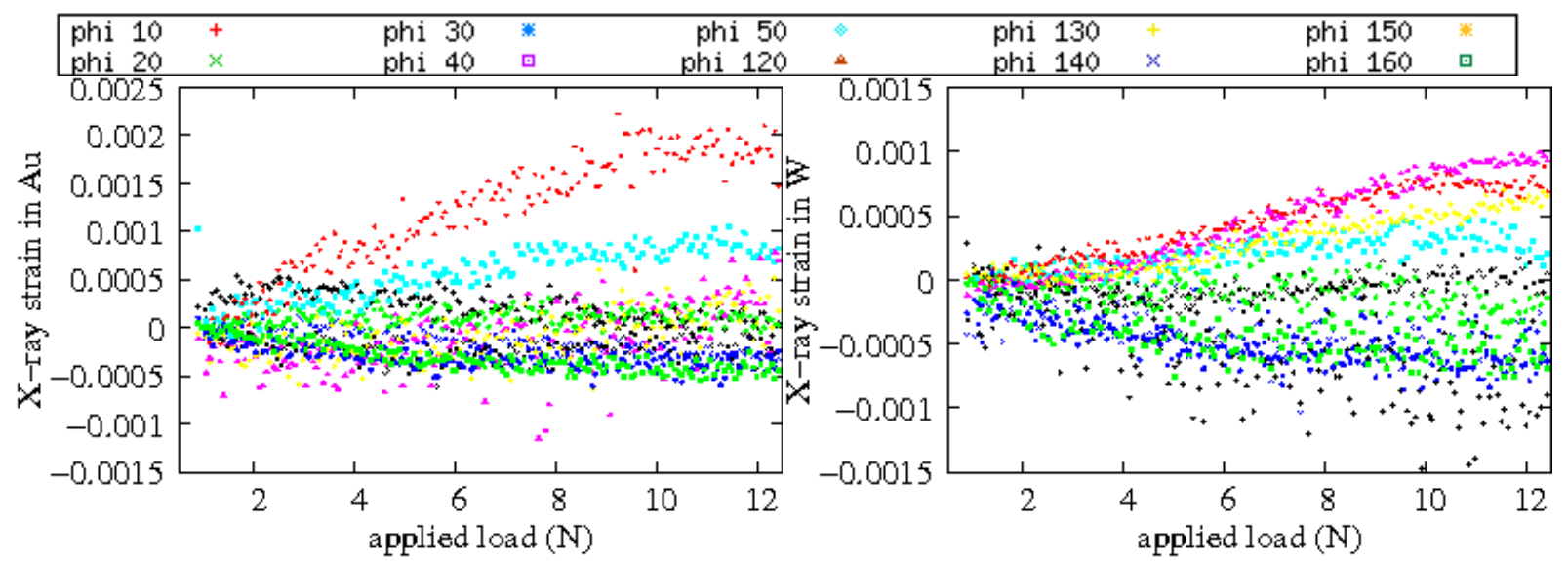

Figure 4. Strain evolution in gold (left) and tungsten (right) for the $12 \mathrm{~nm}$ period sample (with a rotation of $\left.15^{\circ}\right) . \varphi$ positions are the same for both phase.

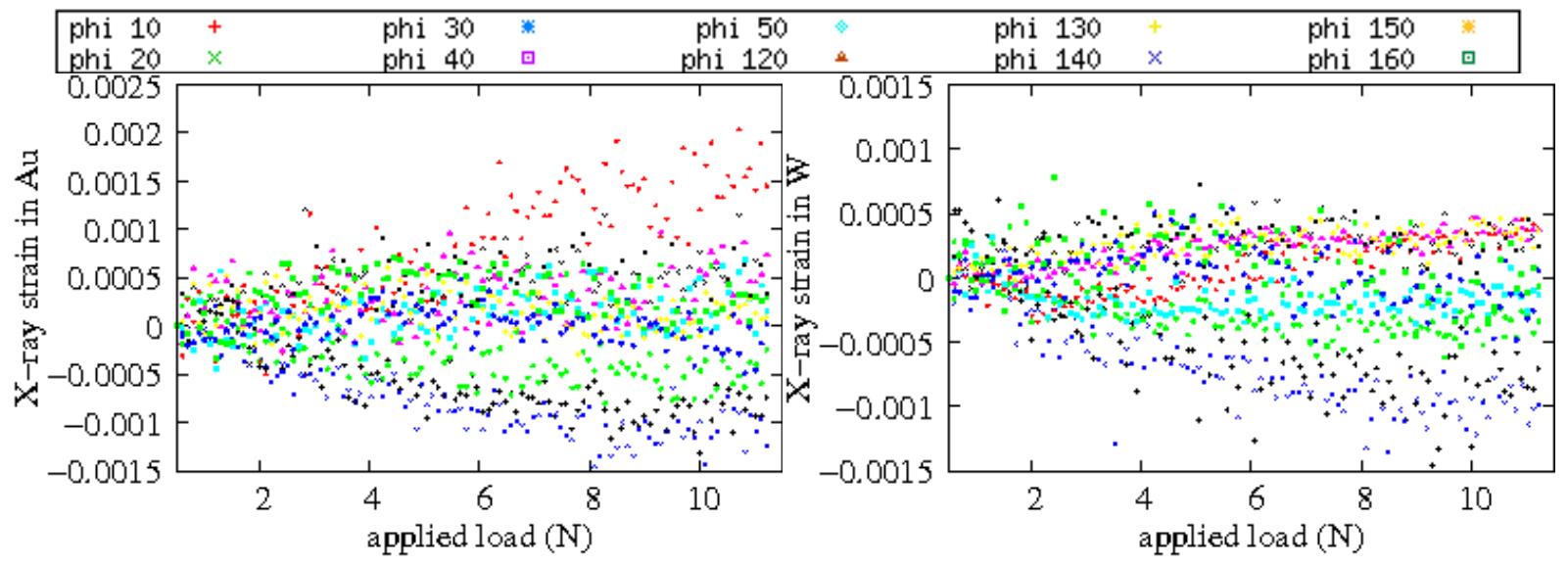

Figure 5. Strain evolution in gold (left) and tungsten (right) for the $6 \mathrm{~nm}$ period sample (with a rotation of $\left.15^{\circ}\right) . \varphi$ positions are the same for both phase.

When the period decreases (i.e. down to 6 and $3 \mathrm{~nm}$ ) peaks become larger and an overlap appears. Figure 5 presents the X-ray strain evolution for the $6 \mathrm{~nm}$ period sample. If we compare with the $12 \mathrm{~nm}$ specimen, we observe that the data points are much more scattered due to the peaks overlapping and the intensity decreases. However, we notice a decrease of the strain value for equivalent $\varphi$ positions between the $12 \mathrm{~nm}$ and $6 \mathrm{~nm}$ period sample which can be related to size effect.

For smaller sample period $(3 \mathrm{~nm})$, we can observe only one peak close to the position of the theoretical tungsten peak. Phase distinction is not possible any more. 


\section{CONCLUSION}

Using a 2D X-ray diffraction detector and dynamical loading, thin films elastic behavior can be studied with a drastically decrease of the experiment time as compared to laboratory experiments. While an experiment in a laboratory takes one week, a similar experiment can be done on synchrotron source within a few hours, with more positions in $\varphi$ and more load steps and thus with expected improved precision. Results show that we can easily follow the behavior of each phase in a multilayer film when the period is large enough (here over $6 \mathrm{~nm}$ ). When the period decreases below this value, peaks overlapping make the analysis more and more difficult.

\section{AKNOWLEDGMENTS}

The authors wish to thank S. Teat for his help in conducting X-ray diffraction measurements at ALS, on the 11.3.1 synchrotron beam line. This work has been granted by the French Agency for Research (ANR) through the Pnano project entitled Cmonano (ANR-05-PNANO-069). The Advanced Light Source is supported by the Director, Office of Science, Office of Basic Energy

Sciences, of the U.S. Department of Energy under Contract No. DE-AC02-05CH11231

\section{REFERENCES}

1. V. B. Shenoy, Phys. Rev. B 71, 2005094104.

2. $\quad$ K. Van Workum, J. J. de Pablo, Phys. Rev. E 67, 2003031601.

3. K. F. Badawi, P. Villain, Ph. Goudeau, P.-O. Renault, Appl. Phys. Lett. 80, 20024705.

4. D. Y. W. Yu, F. Spaepen, J. Appl. Phys. 95, 20042991.

5. A. J. Kalkmann, A. H. Verbruggen, and G. C. A. M. Janssen, Appl. Phys. Lett. 78, 2001 2673.

6. J. Schiøtz, T. Vegge, F. D. Di Tolle, and K. W. Jacobsen, Phys. Rev. B 60, 199911971.

7. $\quad$ M. Jaouen, J. Pacaud, C. Jaouen, Phys. Rev. B 64, 2001, 144106.

8. $\quad$ L.G. Zhou, H. Huang, Appl. Phys. Lett. 86 2004,1

9. I.C. Noyan, G. Sheikh, Mat. Res. Soc. Symp. Proc. 308 1993, 3

10. D. Faurie, O. Castelnau, R. Brenner, P.O. Renault, E. Le Bourhis, Ph. Goudeau, G. Patriarche, Appl. Phys. Lett. 892006061911

11. D. Faurie, P.-O. Renault, E. Le Bourhis, P. Villain, Ph. Goudeau, K. F. Badawi, Thin Solid Films, 469-470 2004201.

12. Yu, Y.H., Lai M.O. Lu L. Zheng G.Y., Surface and Coatings Techn. 20020064006

13. Pina J., Dias A. Lebrun J.L., Mat. Sci. Engin. A 2671999130

14. K.J. Martinschitz, E. Eiper, S. Massl, H. Köstenbauer, R. Daniel, G. Fontalvo, C. Mitterer, J. Keckes, J. Appl. Cryst. 39 (2006) 777

15. J. Böhm, P. Gruber, R. Spolenak, A. Stierle, A. Wanner, E. Arzt, Review of Scientific Instruments 75, (2004) 1110.

16. P. Villain, Ph. Goudeau, P.-O. Renault, K. F. Badawi, Appl. Phys. Lett. 81, (2002) 4365.

17. A. Hammersley, fit2d software, http://www.esrf.eu/computing/scientific/FIT2D/

18. Faurie, D., Renault, P.-O., Le Bourhis, E., Goudeau, P, Materials Research Society Symposium Proceedings 875, art. no. O4.7, 2005, 103-108 